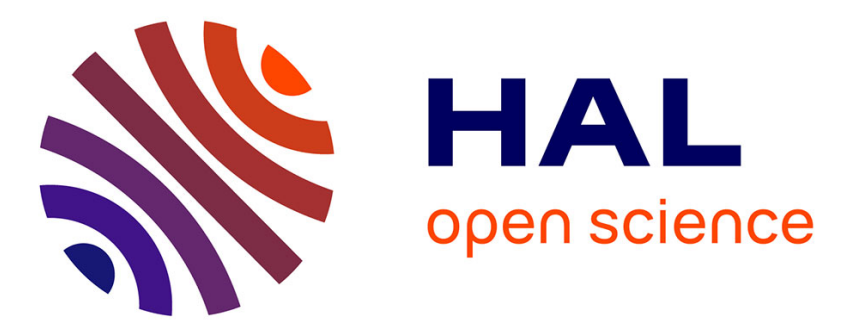

\title{
Accurate 3D Lighthouse Localization of a Low-Power Crystal-Free Single-Chip Mote
}

\author{
Brian Kilberg, Felipe M R Campos, Filip Maksimovic, Thomas Watteyne,
} Kristofer S J Pister

\section{- To cite this version:}

Brian Kilberg, Felipe M R Campos, Filip Maksimovic, Thomas Watteyne, Kristofer S J Pister. Accurate 3D Lighthouse Localization of a Low-Power Crystal-Free Single-Chip Mote. Journal of Microelectromechanical Systems, 2020, 29 (5), 10.1109/JMEMS.2020.3011460 . hal-02916806

\section{HAL Id: hal-02916806 https://hal.inria.fr/hal-02916806}

Submitted on 18 Aug 2020

HAL is a multi-disciplinary open access archive for the deposit and dissemination of scientific research documents, whether they are published or not. The documents may come from teaching and research institutions in France or abroad, or from public or private research centers.
L'archive ouverte pluridisciplinaire HAL, est destinée au dépôt et à la diffusion de documents scientifiques de niveau recherche, publiés ou non, émanant des établissements d'enseignement et de recherche français ou étrangers, des laboratoires publics ou privés. 


\title{
Accurate 3D Lighthouse Localization of a Low-Power Crystal-Free Single-Chip Mote
}

\author{
Brian G. Kilberg, Student Member, IEEE, Felipe M. R. Campos, Student Member, IEEE, Filip Maksimovic, \\ Thomas Watteyne, Senior Member, IEEE, Kristofer S. J. Pister
}

\begin{abstract}
We present a system for centimeter-precision 3 dimensional localization of a $2 \times 3 \times 0.3 \mathrm{~mm}^{3}, 5 \mathrm{mg}$, wireless systemon-chip by utilizing a temporally-structured infrared illumination scheme generated by a set of base stations. This 3D localization system builds on previous work by adding a second lighthouse station to enable 3D localization and using the integrated wireless radio, making the localization system fully wireless. We demonstrate 3D tracking with mean absolute errors of $1.54 \mathrm{~cm}, 1.50 \mathrm{~cm}$, and $5.1 \mathrm{~cm}$ for the $X, Y$, and $Z$ dimensions. This is the first time such a lighthouse localization system has been able to localize a monolithic single-chip wireless system.
\end{abstract}

Index Terms-tracking, laser applications, state estimation, stereo vision, microcontrollers, position measurement

\section{INTRODUCTION}

Autonomous microsystems, such as microrobots, have limited payload capabilities, which require their processing and communication platforms to be highly miniaturized. The $2 \times 3 \times 0.3 \mathrm{~mm}^{3}, 5 \mathrm{mg}$, Single Chip Micro Mote (SC $\left.\mu \mathrm{M}\right)$ [1] is an example of a suitable system for microrobots, containing an ARM Cortex-M0 microprocessor and a crystal-free $2.4 \mathrm{GHz}$ radio with full IEEE 802.15.4 compatibility and limited Bluetooth Low Energy transmit capabilities. Localization of these systems is important for them to perform intelligent tasks in the real world. One promising localization system for $\mathrm{SC} \mu \mathrm{M}$ is "lighthouse" localization, a type of optical localization with millimeter-scale precision that uses horizontally and vertically rotating planar laser sweeps and omnidirectional synchronization pulses. These pulses allow an object to determine its azimuth and elevation angles relative to a lighthouse base station [2], [3].

The tracked object in this system requires an infraredsensitive photodiode that detects the lighthouse station's laser sweeps. In [4], we demonstrated that SC $\mu \mathrm{M}$ 's integrated optical receiver, originally intended for contact-free optical programming, can be repurposed to accurately detect the laser sweeps generated by an HTC Vive lighthouse base station $^{1}$, which enables calculation of the mote's azimuth and elevation angles relative to the lighthouse. This work further develops this application and demonstrates the full 3D tracking of a $\mathrm{SC} \mu \mathrm{M}$ chip, while using its on-board radio to wirelessly communicate its position. This work enables the ability to localize, with centimeter accuracy, a fully-monolithic

B. Kilberg, F. Campos, F. Maksimovic, K. Pister are with the Berkeley Sensor and Actuator Center, University of California, Berkeley, CA, 94720 USA. e-mail: ( $\{$ bkilberg,fmrcampos,fil,ksjp $\}$ @ berkeley.edu).

T. Watteyne is with Inria, Paris, France. e-mail: thomas.watteyne@inria.fr

${ }^{1}$ https://www.vive.com/eu/accessory/base-station/

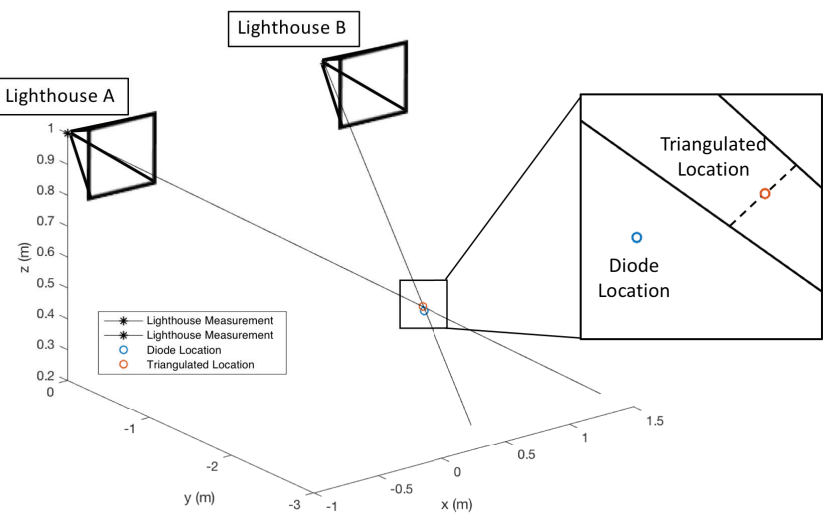

Fig. 1. Triangulation of lighthouse measurement rays. The relative angle measurements from each lighthouse create rays pointing in the direction of the mote. These projected rays are unlikely to cross due to measurement noise, so the direct linear transform is used to estimate their intersection and triangulate the SC $\mu \mathrm{M}$ 's position.

microsystem without compromising system form factor, which is important and beneficial for a variety of applications such as microrobotics, wireless sensor networks, and other MEMSbased systems.

\section{The Single Chip Micro Mote (SC $\mu \mathrm{M})$}

The Single Chip Micro Mote (SC $\mu \mathrm{M})$, shown in Fig. 2, is a fully monolithic wireless System-on-a-Chip developed for microbotic applications [1]. Intended for payload-constrained applications like micro-robotics, the $2 \times 3 \times 0.3 \mathrm{~mm}^{3} \mathrm{SC} \mu \mathrm{M}$ chip does not require any external connections except for a power source and an antenna. For example, $\mathrm{SC} \mu \mathrm{M}$ has been demonstrated driving an electrostatic inchworm-motorpowered microrobotic gripper [5]. Additionally, Chang et al. successfully demonstrated $\mathrm{SC} \mu \mathrm{M}$ running a low-power timesynchronized network protocol $(6 \mathrm{TiSCH})$ and participating in a time-synchronized channel hopping mesh network [6].

$\mathrm{SC} \mu \mathrm{M}$ features an integrated optical receiver, initially designed for contact-free optical programming [4]. The active power of the optical receiver is $1.5 \mu \mathrm{W}$, compared to the active power of the entire system, which can be up to $2 \mathrm{~mW}$ [4].

\section{Lighthouse TRIANGULATION}

\section{A. Base Principle}

Lighthouse-based localization was initially developed for localizing constrained low-power electronic objects [2]. In 


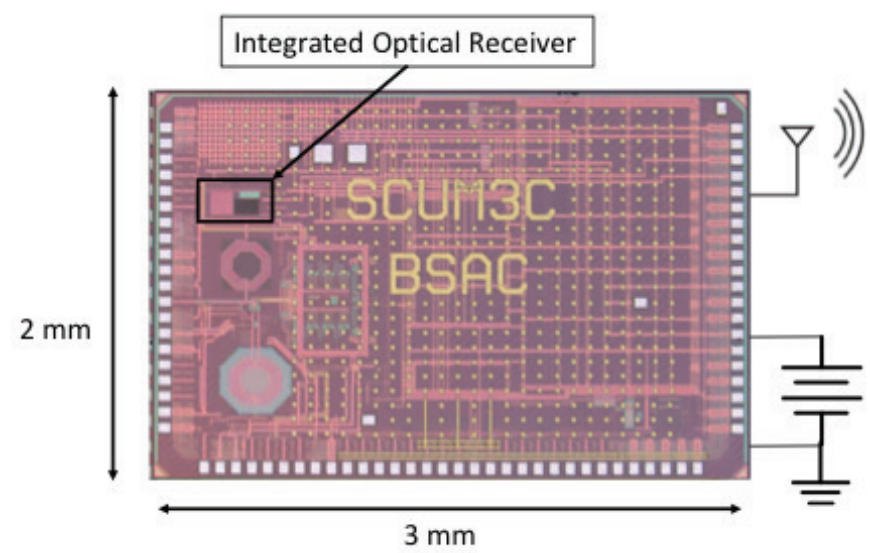

Fig. 2. Die photo of the Single Chip Micro Mote $(\mathrm{SC} \mu \mathrm{M})$. The integrated optical receiver was initially designed for contact-free optical programming. We re-purpose it to allow the mote to localize itself by timing the horizontal and vertical laser scans from a HTC Vive lighthouse localization system. Only the three electrical connections shown are necessary for this application.

recent years, it has been applied for precisely and quickly measuring the position and orientation of Virtual Reality (VR) headsets such as the HTC Vive [3]. The base principle is that a lighthouse base station (which is typically the size of a coffee cup) is equipped with a laser which sweeps a laser plane across space, first horizontally, then vertically. An object equipped with a photodiode timestamps the moments it detects the pulse from the horizontal and vertical sweeps. Knowing the speed of those sweeps, it can compute its azimuth and elevation angles, and hence knows it is located on a ray relative to the lighthouse base station.

To obtain a 3D localization, at least two base station are needed, as depicted in Fig. 1. These lighthouses are connected by a wire which allows them to synchronize their pulses to one another; the role of each lighthouse (Lighthouse 1 or Lighthouse 2) is configured by a slider on each lighthouse. Fig. 3 shows a chronogram of the activity of each lighthouse. The lighthouses are equipped with two types of light sources: a high-powered omnidirectional infrared LED (used for sending sync pulses), and two lasers pointed at either of two mirrors that rotate at $120 \mathrm{~Hz}$, at a 90 degree angle. The mirrors are used to sweep through space either in the azimuth or elevation, causing $\mathrm{SC} \mu \mathrm{M}$ to receive a laser pulse. The lighthouses alternate between sending sync pulses and sweeping one of their lasers. Lighthouse 1 always sends a sync pulse before Lighthouse 2. The duration of the sync pulse indicates what the lighthouse will do, per Table I: sweep its azimuth laser, sweep its elevation laser, or keep its lasers off. The duration before receiving the laser pulse encodes the angle to the lighthouse. After receiving an azimuth and elevation pulse, $\mathrm{SC} \mu \mathrm{M}$ can compute on what measurement ray it is relative to the lighthouse that send the pulses. Because of measurement inaccuracies, the measurement rays from both lighthouse base station most likely don't intersect. The challenge is hence to compute the position of the point in 3D space which minimize the distance to both rays.

\begin{tabular}{|l|r|l|}
\hline Pulse Type & Duration & Description \\
\hline$T_{A}$ & $62.5 \mu \mathrm{s}$ & sync pulse announcing azimuth sweep \\
\hline$T_{E}$ & $72.9 \mu \mathrm{s}$ & sync pulse announcing elevation sweep \\
\hline$T_{S}$ & $104.0 \mu \mathrm{s}$ & sync pulse announcing skip (no sweep) \\
\hline$T_{\text {sweep }}$ & $8.3 \mathrm{~ms}$ & full 180 degree sweep of the laser \\
\hline
\end{tabular}

TABLE I

DURATION OF THE PULSE ACTIVITY OF AN HTC ViVE Lighthouse.

\section{B. Receiving Lighthouse Laser Pulses on SC $\mu M$}

As described in [4], $\mathrm{SC} \mu \mathrm{M}$ is able to detect the pulse from an HTC Vive Lighthouse using its optical receiver. We develop custom firmware ${ }^{2}$ to process the structured infrared light emitted by the base stations. This firmware uses interrupts to detect, measure, and decode the laser scan timings and sync pulse widths that are received by $\mathrm{SC} \mu \mathrm{M}$ 's optical receiver. The output of the optical receiver is routed to a GPIO output pin, then back into the processor via multiple GPIO pins: one connected to an active high level interrupt and one connected to an active low level interrupt in order to implement an edge sensitive interrupt.

A nearby computer is equipped with an OpenMote, a popular IEEE 802.15.4-based platform [7]. The OpenMote is programmed to listen to a particular frequency, and report to the computer the frames it receives. Upon measuring the timings from the laser pulses, the $\mathrm{SC} \mu \mathrm{M}$ chip reports those values to the computer over $2.4 \mathrm{GHz}$ IEEE 802.15.4. The Python-based program running on the computer then uses the timings to calculate the relative azimuth and elevation angles between each lighthouse base station and the $\mathrm{SC} \mu \mathrm{M}$ chip.

\section{Lighthouse Projection Model}

A $\mathrm{SC} \mu \mathrm{M}$ chip's 3D location can be calculated by triangulating the directional rays, determined by the azimuth and elevation measurements, from each lighthouse base station. These rays are unlikely to intersect in 3D space due to inherent noise in the measurements, so an estimation method is required to find the triangulation solution that minimizes the error between the two rays (see Fig. 1).

Triangulation is a well-studied problem commonly arising in computer vision with multi-perspective cameras [8]. In fact, modeling each lighthouse base station as a camera allows for both calibration of relative poses of each lighthouse in addition to the use of triangulation methods like the Direct Linear Transform (DLT) for 3D localization [8], [9]. The mathematical model that describes the transformation between a point in 3D global coordinates to a $2 \mathrm{D}$ point on the image plane of a pinhole camera is described by (1).

$$
X_{\text {image }}=\left[\begin{array}{c}
x^{i m g} \\
y^{i m g} \\
1
\end{array}\right]=P X_{\text {global }}
$$

Eq. (2) is the definition of the camera projection matrix, which projects the $3 \mathrm{D}$ object onto the $2 \mathrm{D}$ camera's image plane. $K$ is the matrix representing the intrinsic camera

\footnotetext{
2 available at https://github.com/PisterLab/scum-test-code.
} 


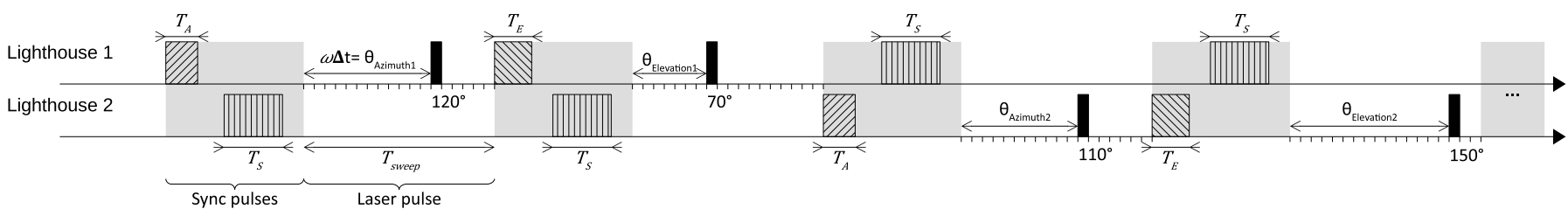

Fig. 3. Chronogram of the pulses received by the $\mathrm{SC} \mu \mathrm{M}$ chip from the two lighthouses. The lighthouses are synchronized over a wired interface. They alternate between periods when sending omnidirectional sync pulses, and periods when sending laser pulses. Lighthouse 1 always sends a sync pulse before Lighthouse 2. The duration of the sync pulse indicates what the lighthouse will do, per Table I. The duration before receiving the laser pulse encodes the angle to the lighthouse. In this illustration, $\mathrm{SC} \mu \mathrm{M}$ measures 120 degrees azimuth / 70 degrees elevation from Lighthouse 1, 110 degrees azimuth / 150 degrees elevation from Lighthouse 2 .

parameters, which are the focal length $f_{x}, f_{y}$ (the distance between the focal plane and the pin hole) and the principal point offset $\left(c_{x}, c_{y}\right)$, which is the offset between the center of the image plane and the pinhole. $K$ is expressed in (3).

$$
\begin{gathered}
P=K[R \mid t] \\
K=\left[\begin{array}{ccc}
f_{x} & 0 & c_{x} \\
0 & f_{y} & c_{y} \\
0 & 0 & 1
\end{array}\right]
\end{gathered}
$$

The pose matrix $[R \mid t]$, expressed in (4), represents the transformation to rotate and translate points from the global frame to the camera frame. The rotation from global to camera frame is represented by $R$, which is composed of $r_{i j}$. The translation from the global frame to the camera frame, in camera frame coordinates, is represented by $t_{i}$.

$$
[R \mid t]=\left[\begin{array}{llll}
r_{11} & r_{12} & r_{13} & t_{1} \\
r_{21} & r_{22} & r_{23} & t_{2} \\
r_{31} & r_{32} & r_{33} & t_{3}
\end{array}\right]
$$

The global point being projected onto the camera's image plane $X_{\text {global }}$ is expressed in (5). It contains the global point in augmented homogeneous representation [8].

$$
X_{\text {global }}=\left[\begin{array}{c}
x \\
y \\
z \\
1
\end{array}\right]
$$

In our triangulation implementation, we model the lighthouse base stations as a pinhole camera, where the center of rotation of the lighthouse laser scans is the pinhole and the intrinsic matrix is $I$. The azimuth and elevation measurements (range $0-\pi$, with $\frac{\pi}{2}$ corresponding to the laser perpendicular to the image plane) are projected at unit distance onto this plane using (6). Fig. 4 illustrates this model.

$$
X_{\text {image }}=\left[\begin{array}{c}
x^{i m g} \\
y^{i m g} \\
1
\end{array}\right]=\left[\begin{array}{c}
\tan \left(\theta_{\text {azimuth }}-\frac{\pi}{2}\right) \\
\tan \left(\theta_{\text {elevation }}-\frac{\pi}{2}\right) \\
1
\end{array}\right]
$$

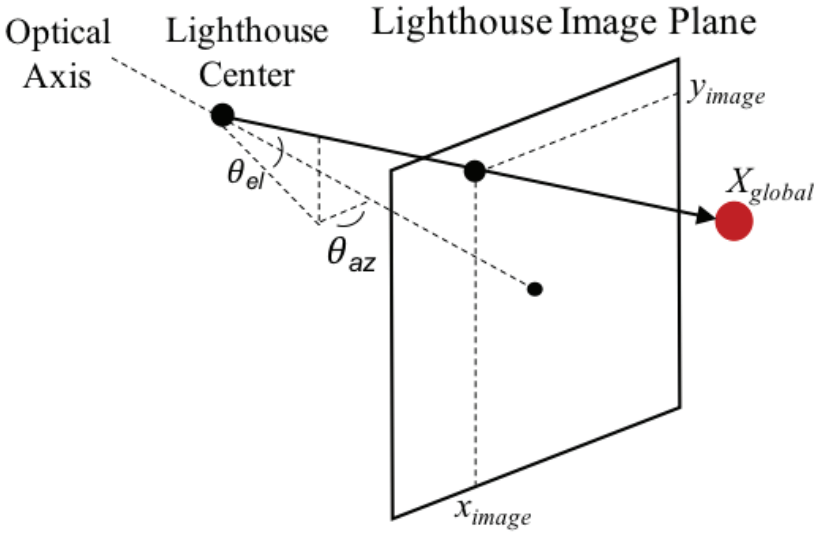

Fig. 4. Camera model of a lighthouse base station. The azimuth and elevation measurements are projected onto the virtual unit distance image plane of the lighthouse.

\section{Triangulation}

We use the Direct Linear Transform (DLT) [8] to triangulate the position of the chip using the relative angle measurements from each lighthouse base station. In the DLT, a system of equations, derived from (7) is set up using the camera projection matrices of each base station from the unknown global point. This system is represented by the matrix $A$ in (8), where $\mathbf{p}^{j}$ is the $j^{\text {th }}$ column of each base station's camera projection matrix. The projection matrix and image coordinates of the second lighthouse base station are denoted $P^{\prime}$ and $x^{\prime}, y^{\prime}$. This system of equations, $A X=0$, is solved using least squares ${ }^{3}$. The computational complexity added by performing triangulation with two lighthouse stations is dominated by this least squares computation involving the $4 \times 4 A$ matrix. Currently, this is calculated on a computer that is receiving $\mathrm{SC} \mu \mathrm{M}$ packets with lighthouse data (the computation requires $10 \mu \mathrm{s}$ on a $2.2 \mathrm{GHz}$ Intel Core i7 processor); further work is required to determine the feasibility of computing this on $\mathrm{SC} \mu \mathrm{M}$. The most recent of each azimuth and elevation measurements are used in the triangulation algorithm. Unfortunately this can lead to inaccuracies if one of these measurements are missed as an out-of-date measurement is then used in its place.

$$
P X=\left[\begin{array}{lll}
x_{\text {image }} & y_{\text {image }} & 1
\end{array}\right]^{T}
$$

\footnotetext{
${ }^{3}$ available at https://github.com/PisterLab/scum_lighthouse_localization.
} 


$$
A=\left[\begin{array}{c}
x \mathbf{p}^{3 T}-\mathbf{p}^{1 T} \\
y \mathbf{p}^{3 T}-\mathbf{p}^{2 T} \\
x^{\prime} \mathbf{p}^{\prime 3 T}-\mathbf{p}^{\prime 1 T} \\
y^{\prime} \mathbf{p}^{\prime 3 T}-\mathbf{p}^{\prime 2 T}
\end{array}\right]
$$

\section{E. Calibration of Lighthouse Projection Matrices}

Using the Direct Linear Transform (DLT) to triangulate the $3 \mathrm{D}$ position of $\mathrm{SC} \mu \mathrm{M}$ in the lighthouse-camera model requires knowing both the intrinsic and extrinsic parameters of the two lighthouse base stations, corresponding to the matrices $K$ and $[R \mid t]$, respectively. Since we know $K$ to be the identity matrix $I$, the calibration task amounts to estimating the extrinsic parameters for each lighthouse in the lighthousecamera model. In computer vision, this is known as the Perspective- $n$-Point problem (or just $\mathrm{P} n \mathrm{P}$ ) [10], whose aim is to determine a camera's pose (position and orientation) given its intrinsic parameters and a set of $n \geq 3$ correspondences between global 3D points and their $2 \mathrm{D}$ projections onto the image plane [11].

For the purpose of this experiment, we build a sizeable set of 3D-2D correspondences: we isolate approx. 1000 sample points of the $\mathrm{SC} \mu \mathrm{M}$ angle data projected onto the unit distance image plane, and their corresponding ground truth $3 \mathrm{D}$ position data. We use the nonlinear Levenberg-Marquardt iterative optimization algorithm to estimate a solution to the $\mathrm{P} n \mathrm{P}$ problem [11]. The Levenberg-Marquardt algorithm attempts to find a solution $[\hat{R} \mid \hat{t}]$ to (9) by minimizing the residual sum of squared distances between the observed projections $\left[x_{i}^{i m g} y_{i}^{i m g} 1\right]^{T}$ and the projected ground truth points $[\hat{R} \mid \hat{t}]\left[\begin{array}{llll}x & y & z & 1\end{array}\right]^{T}$.

$$
\left[\begin{array}{ccc}
x_{1}^{i m g} & \ldots & x_{n}^{i m g} \\
y_{1}^{i m g} & \ldots & y_{n}^{i m g} \\
1 & \ldots & 1
\end{array}\right]=[\hat{R} \mid \hat{t}]\left[\begin{array}{cccc}
x_{1} & x_{2} & \ldots & x_{n} \\
y_{1} & y_{2} & \ldots & y_{n} \\
z_{1} & z_{2} & \ldots & z_{n} \\
1 & 1 & \ldots & 1
\end{array}\right]
$$

To implement our calibration procedure, we used OpenCV's solvePnPRansac method with the CV_ITERATIVE flag [12]. This uses the random sample consensus (RANSAC) iterative method on top of the standard OpenCV LevenbergMarquardt optimization $\mathrm{P} n \mathrm{P}$ solver to make the projection robust to outliers in $\mathrm{SC} \mu \mathrm{M}$ 's angle measurements. We apply the calibration to each lighthouse to recover their relative poses. Fig. 5 illustrates the results of using our calibration procedure by applying the pose of Lighthouse 1 that minimizes the reprojection error.

\section{RESULTS}

We evaluate the accuracy of the $\mathrm{SC} \mu \mathrm{M}$ lighthouse tracking system using the OptiTrack motion capture system, which has sub-mm accuracy. We move the $\mathrm{SC} \mu \mathrm{M}$ chip along a trajectory by hand, while tracking it with both the lighthouse system and the OptiTrack (we use the same methodology as in [4]). The SC $\mu \mathrm{M}$ chip was mounted on a board that had optical tracking markers attached. We make sure to synchronize the OptiTrack's infrared exposure pulses with the lighthouse sync pulses to avoid interference.
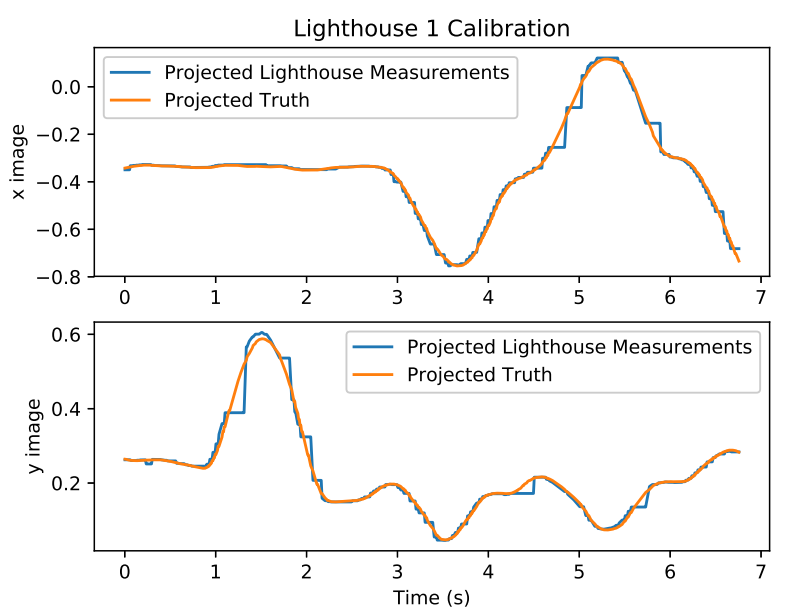

Fig. 5. Illustration of lighthouse calibration. The calibration process uses the projected measurements and a small portion of the OptiTrack truth trajectory data (this data was not used to calculate measurement accuracy). This plot compares the projected lighthouse image points to the ground truth points projected to the lighthouse image plane using the calibrated projection matrix for Lighthouse 1.

Fig. 6 shows the lighthouse-relative azimuth and elevation angles measured during this experiment by each mote, compared to the ground truth angles. Fig. 7 shows the error of the azimuth and elevation tracking; the interquartile range of the measurements is under 1 degree for three of the four angles. The RMS tracking error statistics are skewed by the presence of a few significant errors of up to 10 degrees. These high error points are observed to be more frequent when the OptiTrack system is active, indicating that the OptiTrack's infrared cameras are likely interfering with the lighthouse signal despite the synchronization protocol. When clear outliers are removed in post-processing (error $>10$ degrees), the RMS tracking error is $0.63 \mathrm{deg}, 0.37 \mathrm{deg}, 0.60 \mathrm{deg}$, and $3.87 \mathrm{deg}$. The angular resolution could be limited by optical receiver jitter; preliminary data show up to $\pm 5 \mu$ s of variation in lighthouse pulse timing, corresponding to \pm 0.2 degrees of variation. Fig. 8 shows the $3 \mathrm{D}$ triangulated tracking data for the experiment, compared to ground truth. The mean absolute error for $3 \mathrm{D}$ tracking is $1.54 \mathrm{~cm}, 1.50 \mathrm{~cm}$, and $5.1 \mathrm{~cm}$ for the $\mathrm{X}, \mathrm{Y}$, and $\mathrm{Z}$ dimensions. The $\mathrm{Z}$ dimension represents the distance between the lighthouses and the SC $\mu \mathrm{M}$ mote, so it's triangulation error is larger because of the short $0.5 \mathrm{~m}$ baseline between the two lighthouse stations.

We characterize the operating range of the $\mathrm{SC} \mu \mathrm{M}$ lighthouse over distance from lighthouse and incidence angle of the $\mathrm{SC} \mu \mathrm{M}$ mote relative to the lighthouse pulses. Specifically, we measure the percent of lighthouse measurements received at various distances from a lighthouse base station (Fig. 10), with the SC $\mu \mathrm{M}$ mote directly in front of the base station. The maximum effective range of the localization system is $1 \mathrm{~m}$. We measure the effect of the angle of incidence on reliability by rotating the $\mathrm{SC} \mu \mathrm{M}$ mote in a stationary position directly in front of two adjacent lighthouse base stations (Fig. 11). The maximum angle the $\mathrm{SC} \mu \mathrm{M}$ mote could be rotated with respect to the lighthouses is $70^{\circ}$. As $\mathrm{SC} \mu \mathrm{M}$ 's 

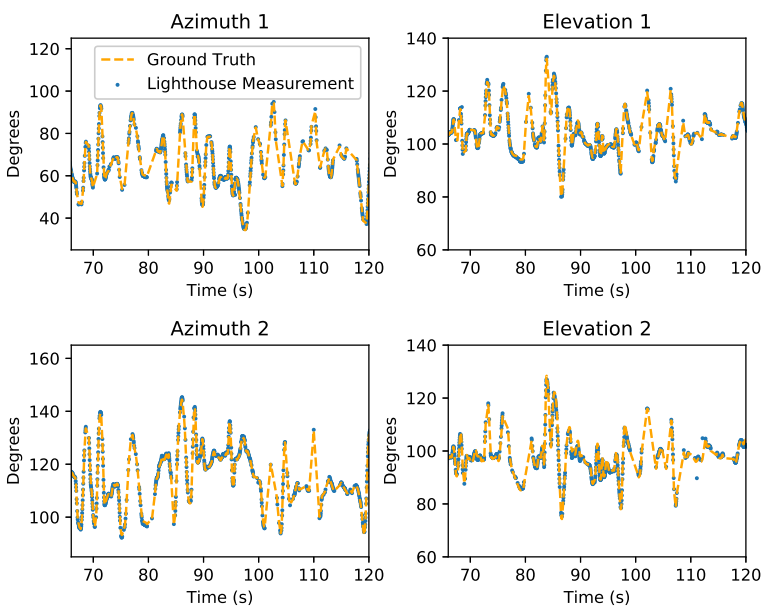

Fig. 6. Experimental azimuth and elevation measurements of a $\mathrm{SC} \mu \mathrm{M}$ mote compared to motion capture ground truth. The lighthouse-relative ground truth azimuth and elevation angles were determined by using the calibrated lighthouse poses to project the 3-dimensional world-frame ground truth trajectory to 2-dimensional lighthouse-relative azimuth and elevation angles.

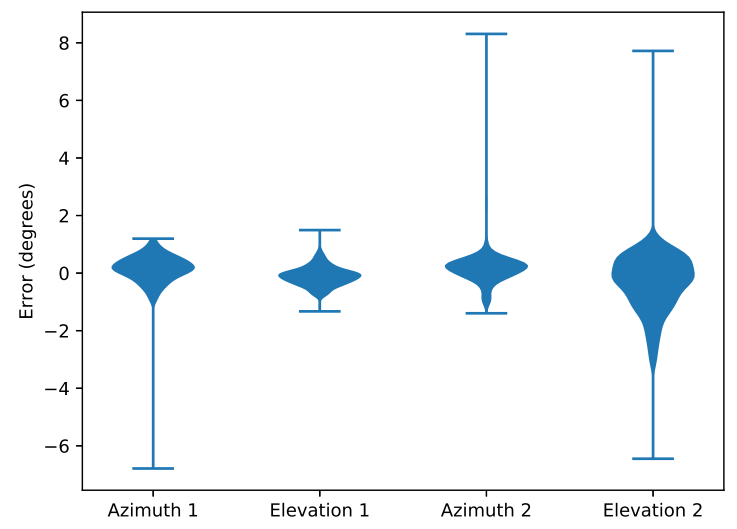

Fig. 7. Violin plot showing distribution of azimuth and elevation angle error. One outlier data point that is 80 degrees from the mean of the elevation 2 data is not shown. The majority of error points are within a tight distribution, with only a few points far from the mean. The RMS tracking error, with the 80 degree outlier removed, is $0.63 \mathrm{deg}, 0.37 \mathrm{deg}, 0.60 \mathrm{deg}$, and $3.87 \mathrm{deg}$ for azimuth 1 , elevation 1 , azimuth 2 , and elevation 2 , respectively.

optical receiver is designed for optical programming and not lighthouse localization, a future redesign of the receiver system should increase the detection range of the lighthouse signal.

\section{FUTURE WORK}

While these results are promising, several problems limit this system's utility. First, intermittent outliers with significant error ( $>10$ degrees) are present. Second, this system relies on line-of-sight and is vulnerable to occlusion of the lighthouse base stations. Third, the diminished lighthouse reception rate over distance and incidence angle can intermittently reduce the triangulation rate significantly. Finally, out-of-date measurements can cause inaccuracies in the triangulation algorithm, which relies on four measurements that do not occur simultaneously.
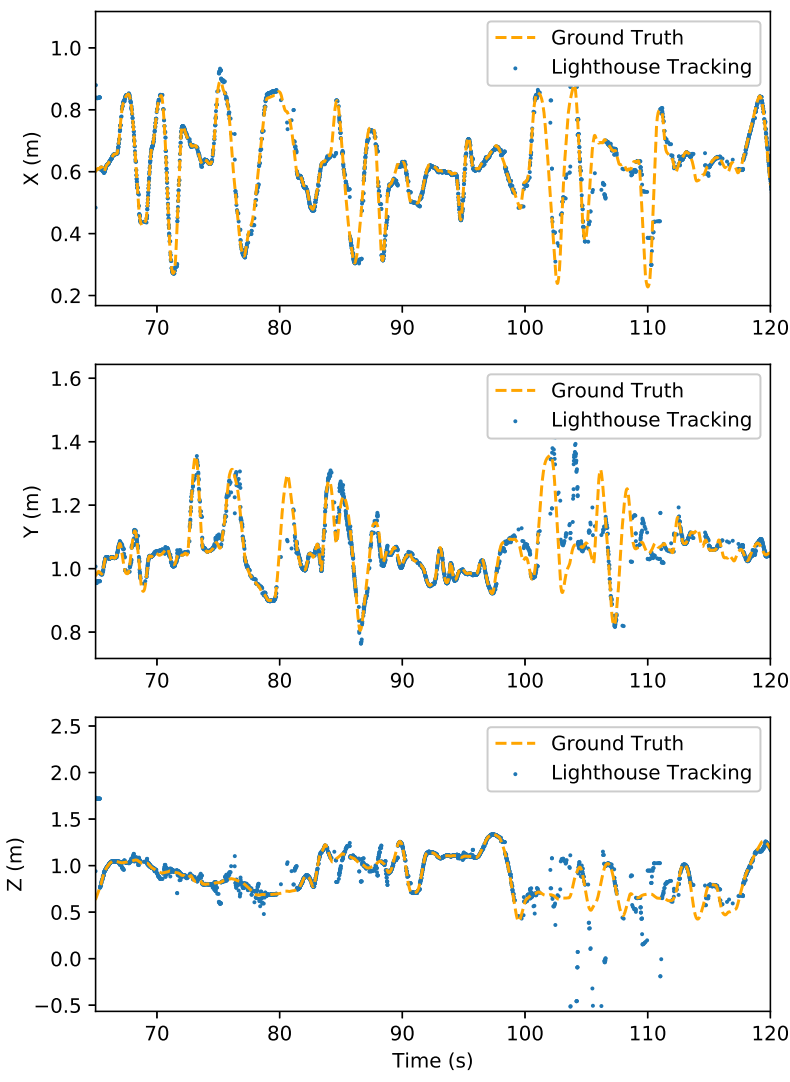

Fig. 8. Triangulation of lighthouse data to reconstruct the trajectory of the SC $\mu \mathrm{M}$ chip. Large deviations in tracking are caused by missed measurements. In our system, a missed measurement gets replaced by the previous measurement. Significantly out-of-date measurements can resulting in inaccurate triangulation. The subset of data used for lighthouse calibration is excluded from this data.

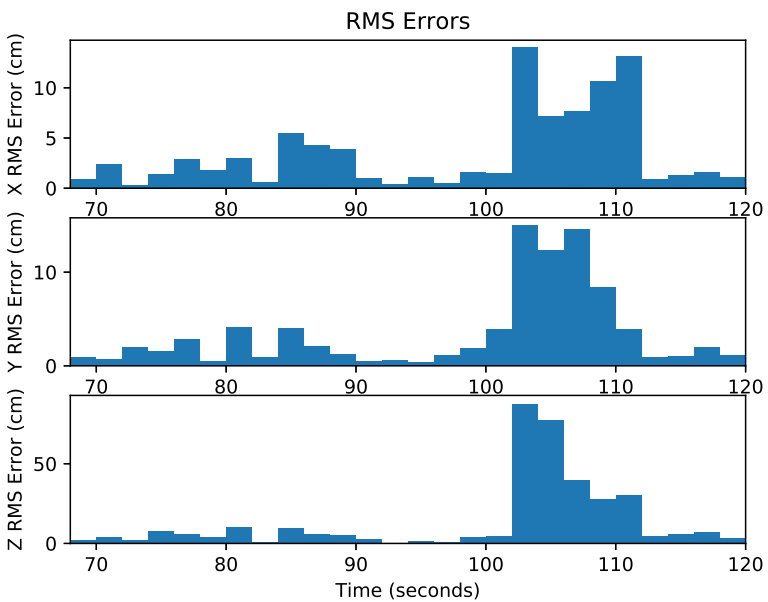

Fig. 9. Triangulation error of lighthouse tracking over time. The RMS tracking error is characterized by periods of time with minimal error and periods of time with significant error. Periods of significant error can be caused by line-of-sight occlusion, infrared interference, or RF interference events. These periods are observed more frequently when the OptiTrack system is active, indicating that IR interference could be the cause. 


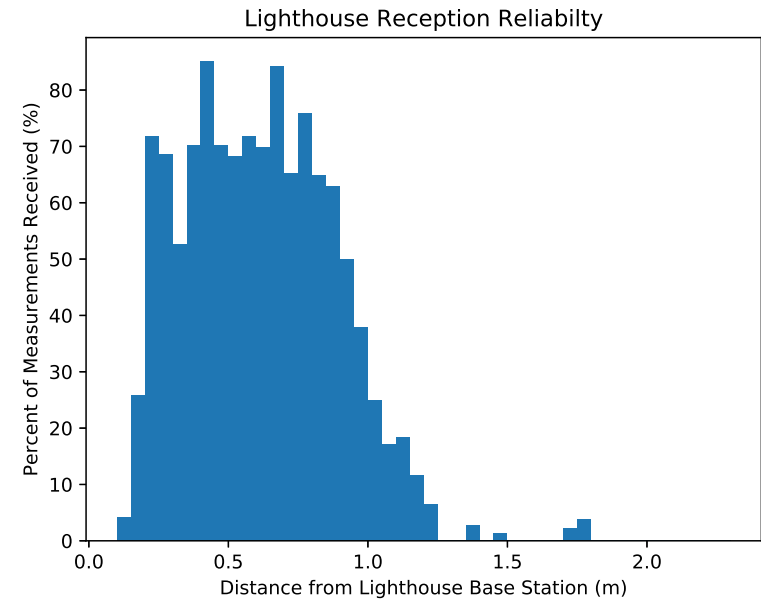

Fig. 10. Percent of lighthouse measurements received, as a function of distance to lighthouse. The reliability of the lighthouse measurements drops off at around $1 \mathrm{~m}$. An active motion capture system is used in this experiment to measure $\mathrm{SC} \mu \mathrm{M}$ position, which could have a small effect on the lighthouse reliability due to infrared interference.

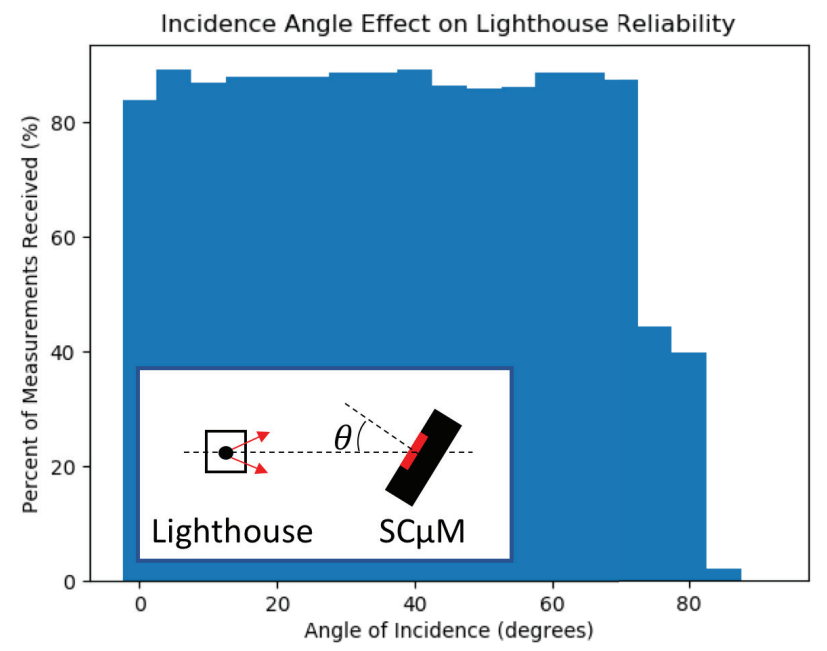

Fig. 11. Effect of incidence angle $(\theta)$ on reliability of lighthouse measurements. An angle of $0^{\circ}$ corresponds to the face of the $\mathrm{SC} \mu \mathrm{M}$ parallel to the face of the lighthouse base station, with the laser scans perpendicular to the face of SC $\mu \mathrm{M}$. A rapid dropoff in lighthouse measurement reliability occurs when $\mathrm{SC} \mu \mathrm{M}$ is rotated $>70^{\circ}$ relative to the lighthouses. In this experiment, the lighthouses are $9 \mathrm{~cm}$ apart, the $\mathrm{SC} \mu \mathrm{M}$ was $47 \mathrm{~cm}$ away, centered in between the two lighthouses. The incidence angles are measured manually, rather than by a motion capture system.

These problems could be solved by fusing the lighthouse measurements with inertial measurement unit (IMU) data. Sensor fusion algorithms like the Extended Kalman Filter (EKF) could use statistical measures such as the Mahalanobis distance to reject outlier lighthouse measurements [13], [14]. Furthermore, during lighthouse occlusion events, this state estimator could still calculate the position of the $\mathrm{SC} \mu \mathrm{M}$ chip by integrating IMU measurements, improving occlusion tolerance. In fact, we demonstrated in [15] the ability of an EKF to provide occlusion tolerance in a lighthouse-IMU sensor fusion system. Sensor fusion would also increase the tracking rate of the system to the IMU's update rate, which could alleviate the aforementioned lighthouse update rate issues. Finally, an EKF, being a non-linear estimator, could calculate position from direct individual lighthouse angle measurements instead of triangulation from four temporally-disparate measurements.

It would also be beneficial to implement the triangulation on-board the $\mathrm{SC} \mu \mathrm{M}$ chip itself, which would improve localization scalability for large numbers of $\mathrm{SC} \mu \mathrm{M}$ chips.

\section{CONCLUSION}

We have demonstrated the 3D localization of a $1.8 \mathrm{~mm}^{3}$ crystal-free wireless SoC using SC $\mu$ M's integrated optical receiver and commercially available virtual reality tracking hardware. This system does not require any extra components on the $\mathrm{SC} \mu \mathrm{M}$ side, thus preserving its miniature form factor. Applications in microrobotics and personal telemetry could be improved by this localization system.

\section{REFERENCES}

[1] F. Maksimovic, B. Wheeler, D. C. Burnett, O. Khan, S. Mesri, I. Suciu, L. Lee, A. Moreno, A. Sundararajan, B. Zhou et al., "A CrystalFree Single-Chip Micro Mote with Integrated 802.15.4 Compatible Transceiver, Sub-mW BLE Compatible Beacon Transmitter, and Cortex M0," in Symposium on VLSI Circuits (VLSI). IEEE, 2019.

[2] K. Römer, "The Lighthouse Location System for Smart Dust," in International Conference on Mobile Systems, Applications and Services (MobiSys). ACM, 2003, pp. 15-30.

[3] M. Borges, A. Symington, B. Coltin, T. Smith, and R. Ventura, "HTC Vive: Analysis and Accuracy Improvement," in IEEE/RSJ International Conference on Intelligent Robots and Systems (IROS). IEEE, 2018, pp. 2610-2615.

[4] B. Wheeler, A. Ng, B. Kilberg, F. Maksimovic, and K. S. J. Pister, "A Low-Power Optical Receiver for Contact-free Programming and 3D Localization of Autonomous Microsystems," in Ubiquitous Computing, Electronics \& Mobile Communication Conference (UEMCON). IEEE, 2019.

[5] A. Moreno, F. Maksimovic, L. Lee, B. Kilberg, C. Schindler, H. Gomez, D. Teal, D. Acker-James, A. Fearing, J. S. Rentmeister, J. T. Stauth, and K. S. J. Pister, "Single-chip micro-mote for microrobotic platforms," in GOMACtech, 2020.

[6] T. Chang, T. Watteyne, B. Wheeler, F. Maksimovic, O. Khan, S. Mesri, L. Lee, I. Suciu, D. Burnett, X. Vilajosana et al., "6tisch on sc $\mu$ m: Running a synchronized protocol stack without crystals," Sensors, vol. 20, no. 7 , p. $1912,2020$.

[7] X. Vilajosana, P. Tuset, T. Watteyne, and K. Pister, "Openmote: Opensource prototyping platform for the industrial iot," in International Conference on Ad Hoc Networks. Springer, 2015, pp. 211-222.

[8] R. Hartley and A. Zisserman, Multiple View Geometry in Computer Vision. Cambridge University Press, 2003.

[9] G. Wetzstein, EE 267 Course Notes: 6-DOF Pose Tracking with the VRDuino. Stanford University, March 2019.

[10] V. Lepetit, F. Moreno-Noguer, and P. Fua, "EPnP: An Accurate O(n) Solution to the PnP Problem," International Journal of Computer Vision, vol. 81, February 2009.

[11] Z. Zhang, "A Flexible New Technique for Camera Calibration," IEEE Transactions on Pattern Analysis and Machine Intelligence, vol. 22, no. 11, pp. 1330-1334, November 2000.

[12] G. Bradski, "The OpenCV Library," Dr. Dobb's Journal of Software Tools, 2000

[13] G. Chang, "Robust Kalman Filtering Based on Mahalanobis Distance as Outlier Judging Criterion," Journal of Geodesy, vol. 88, no. 4, pp. 391-401, 2014.

[14] M. S. Grewal and A. P. Andrews, Kalman filtering: Theory and Practice with MATLAB. John Wiley \& Sons, 2014.

[15] F. M. R. Campos, C. Schindler, B. Kilberg, and K. S. J. Pister, "Lighthouse Localization of Wireless Sensor Networks for LatencyBounded, High-Reliability Industrial Automation Tasks," in International Conference on Factory Communication Systems (WFCS). IEEE, 2020 . 


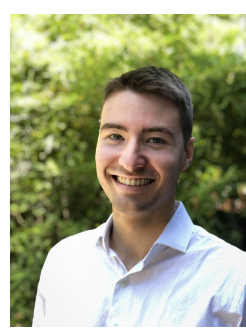

Brian G. Kilberg Brian Kilberg completed his B.S. in Electrical Engineering at the University of Wisconsin-Madison in 2014. He completed a PhD in Electrical Engineering and Computer Sciences from the University of California, Berkeley in 2020. His work focuses on microrobotic systems, low power wireless sensor networks, and resource-constrained localization for robotics.

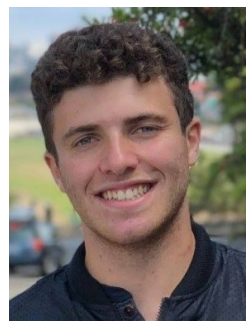

Felipe M. R. Campos Felipe Mulinari Rocha Campos was born in 1999 in Los Angeles, California. He is an undergraduate working towards his Bachelor of Science (B.Sc) in Electrical Engineering \& Computer Science at the University of California, Berkeley in Berkeley, California. He joined the Berkeley Sensor \& Actuator Center's Qualcomm Swarm Lab in 2018, where he has since published four scientific papers in the realm of localization, robotic automation, and Internet of Things. Previously, he worked at the University of Wisconsin-Madison's Wisconsin IceCube Particle Astrophysics Center, where he published three scientific papers on distributed sensing of cosmic rays and other energetic particles. $\mathrm{He}$ is currently interning at Google, and has previously interned at Microsoft working on experimental projects.

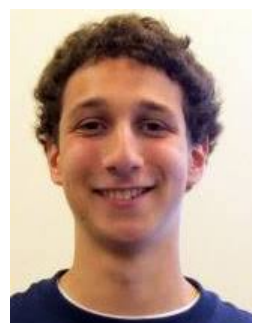

Filip Maksimovic Filip Maksimovic received the B.S. degree in electrical engineering and aerospace engineering from the University of Colorado at Boulder and the Ph.D. degree in electrical engineering and computer sciences from the University of California at Berkeley, Berkeley, in 2018. His research interests include low-power radio frequency communication and wireless sensor networks.

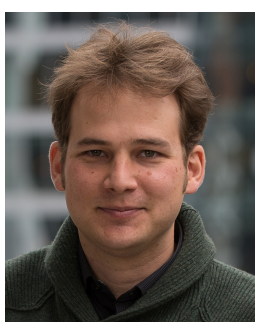

Thomas Watteyne Thomas Watteyne (http://www.thomaswatteyne.com/,

@ thomaswatteyne) is an insatiable enthusiast of low-power wireless mesh technologies. He holds an Research Director position at Inria in Paris, in the EVA research team, where he leads a team that designs, models and builds networking solutions based on a variety of Internet-of-Things (IoT) standards. He is Senior Networking Design Engineer at Analog Devices, in the Dust Networks product group, the undisputed leader in supplying low power wireless mesh networks for demanding industrial process automation applications. Since 2013, he co-chairs the IETF 6TiSCH working group, which standardizes how to use IEEE802.15.4e TSCH in IPv6-enabled mesh networks, and is member of the IETF Internet-of-Things Directorate. Prior to that, Thomas was a postdoctoral research lead in Prof. Kristofer Pister's team at the University of California, Berkeley. He founded and co-leads Berkeley's OpenWSN project, an open-source initiative to promote the use of fully standards-based protocol stacks for the IoT. Between 2005 and 2008, he was a research engineer at France Telecom, Orange Labs. He holds a PhD in Computer Science (2008), an MSc in Networking (2005) and an MEng in Telecommunications (2005) from INSA Lyon, France. He is Senior member of IEEE. He is fluent in 4 languages.

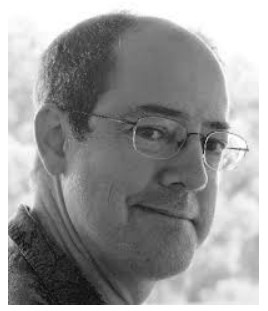

Kristofer S. J. Pister Kristofer Pister is a professor in the UC Berkeley Electrical Engineering and Computer Sciences department. He is faculty director of the Marvell Nanofabrication Laboratory, co-director of the Berkeley Sensor and Actuator Center, and codirector of the Ubiquitous Swarm Lab. He received a BS in applied physics from UC San Diego in 1986 and MS and PhD degrees in EECS from UC Berkeley in 1989 and 1992, then taught in the UCLA electrical engineering department until joining the Berkeley faculty in 1996. His accomplishments include the development of Smart Dust—sub-millimeter wireless sensor network nodes - and he has since worked toward fully autonomous microsystems. 